\title{
Discovering the Traits of Personality in Anesthesiologists at Different Stages of Emotional Burnout Syndrome
}

\author{
Alexey Kokoshko ${ }^{1}$, Nurilya Maltabarova ${ }^{1}$, Aigul Abduldayeva ${ }^{2, *}$, Galiya Smailova $^{3}$ and \\ Nasrulla Shanazarov ${ }^{4}$
}

${ }^{1}$ Department of Ambulance and Anesthesiology - Reanimatology, JSC "Astana Medical University", Beibitshilik Str., 49, 010000 Astana, Kazakhstan

${ }^{2}$ Department of Preventive Medicine and Nutrition, JSC "Astana Medical University", Beibitshilik Str., 49, 010000 Astana, Kazakhstan

${ }^{3}$ Department of Internal Medicine No. 2, JSC "Astana Medical University", Beibitshilik Str., 49, 010000 Astana, Kazakhstan

${ }^{4}$ Department of Surgical Diseases No. 2, JSC "Astana Medical University", Beibitshilik Str., 49, 010000 Astana, Kazakhstan

\begin{abstract}
To date, intensive care medicine, anesthesiology, and resuscitation are medical spheres that deal with lifesaving issues, particularly the treatment of patients in critical or life-threatening situations. The profession of anesthesiologist-resuscitator is relatively new, although it has centuries-old prehistory. The study aims to identify personality traits that predispose to the formation of emotional burnout syndrome in anesthesiologists-resuscitators. The research was performed at the Anesthesiology and Resuscitation Departments of the regional healthcare facilities in Astana and Akmola cities. Findings obtained through this research confirm the hypothesis that personality traits in anesthesiologists-resuscitators are factors that facilitate the development of emotional burnout syndrome. Such a discovery necessitates the prevention of personality destructions as well as the establishment of conditions to reduce them in the case of occurrence. Proper arrangement of the work schedule and a favorable working environment allows preventing the emotional burnout among employees.
\end{abstract}

Keywords: Anesthesiologist-resuscitator, occupational burnout, occupational stress, professional activities of doctors, psychopathological syndrome.

\section{INTRODUCTION}

Intensive therapy, anesthesiology, and resuscitation are concerned with the lifesaving and emergency treatment of patients.

Formerly, the term "emotional burnout" described a state of emotional exhaustion occurring along with the feeling of worthlessness. For the first time, the term 'burnout' ('emotional burnout') was suggested by American psychiatrist Freidenberg in 1974.

Later, the burnout symptoms extended to include the psychosomatic component. Hence, the syndrome became associated with psychosomatic well-being and was considered a pre-disease state [1]. According to the International Classification of Diseases (ICD-10$\mathrm{CM}$ ), the syndrome of emotional burnout (SEB) is coded with Z73.0. The burnout is defined as a type of psychological stress related to life management difficulties.
The development of emotional burnout syndrome is associated with external and internal factors [2]. The external factors include the workplace arrangement, working schedule, and unfavorable working environment.

Emotional burnout occurs mainly among medical professionals and scientists, as shown by the example of physicists [3,4], but also not rare among other professions [5], such as workers of the petroleum industry [6]. Anyway, intense and constant stress loads result in the formation of all signs of emotional burnout [7]. Also, the syndrome has no geographical dependency and is recorded among representatives of various countries worldwide, such as Colombia, France, South Korea, etc. [2,8,9].

Emotional burnout is characterized by stages of increasing severity. Thus, Perlman and Hartman [10] describe burnout as a response to three major symptom categories of stress:

physiological: focusing on physical symptoms;

affective-cognitive: focusing on attitudes and feelings;
*Address correspondence to this author at the Department of Preventive medicine and nutrition, JSC "Astana Medical University", Beibitshilik Str., 49, 010000 Astana, Kazakhstan; Tel: +77014084387;

E-mail: aigulaabduldayeva1@gmail.com 
- behavioral: focusing on symptomatic behaviors (over-depersonalization and low job productivity).

According to Burisch [11,12], the syndrome of emotional burnout develops in several stages:

1. Pursuing success (warning symptoms include exhaustion and expending excessive amounts of energy).

2. Reduced dedication (to co-workers, patients, others in general, and to work; increased sense of entitlement).

3. Emotional reactions (i.e., depression, aggression).

4. Deterioration of cognitive function, motivation, and emotional-social intelligence.

5. Psychosomatic reactions and reduced immunity.

6. Frustration and negative attitudes.

Also, in $[11,12]$, it is reported that strong dependence on work eventually leads to total despair and existential emptiness.

Although most researchers define stress either as a conflict between a person and an environment or as a result of dysfunctional interactions, traditionally, there is no unified definition for describing occupational stress [13-15]. Thus, the term stress often assumes a stressor or any other factor that causes stress [16].

In the classical sense, stress is an adaptive response. Thus, Vladimir Boiko defines burnout as a developed psychological defense mechanism through which a person "shuts off" one's emotions in a traumatic event [17]. Burnout is an acquired stereotype of emotional, often professional, behavior.

According to Boiko $[18,19]$, the SEB is interrelated with stress and, thus, occurs in stages that correlate with Hans Selye's theory of adaptation [20]. Based on these relations, three stages of burnout can be noted:

1. Alarm stage. Symptoms of the stage are the feeling of being exposed to a traumatic event or circumstances, dissatisfaction, frustration, anxiety, and depression.

2. Resistance. Upon realizing the stress, a person tends to avoid the stressor by suppressing own emotional response.
3. Exhaustion. The body's resources are exhausted, provoking the feeling of emptiness, emotional disconnection, depersonalization, and psychosomatic symptoms. This technique has some advantages over its analogs; namely, it measures quite different phenomena, such as the reaction to external and internal factors, ways of psychological protection, or the condition of the nervous system. Quantitative indicators determine the formation degree of each of the burnout phases. First, the dominant symptoms are diagnosed in individual phases and as a whole, and, afterward, the causative factors are determined like professional environment or subjective-personal features. The diagnostics results using this method provide the basis for further work on reducing risk factors of burnout in the professional environment and work with human resources.

Boiko's burnout measurement scale allows establishing a detailed picture of the syndrome and identifying the dominant symptoms. The mentioned measurement method was chosen due to the possibility of establishing the supposed relationship between the three stages of emotional burnout on the one hand and the place of work, work experience, and gender on the other. The obtained data can be interpreted as recommendations for anesthesiologists in determining different stages of their emotional burnout.

Despite the significant interest and intensive research on the matter, opinions regarding the causes and mechanisms of SEB development vary [13]. To assess occupational burnout, most experts recommend using the Maslach Burnout Inventory, which assumes measuring three dimensions of burnout like emotional exhaustion, depersonalization, and personal accomplishment $[21,22]$.

Additional burnout measures include uni- and multidimensional models. The one-factor model suggests that burnout is a state of physical, emotional, and cognitive exhaustion caused by long-term involvement in emotionally demanding situations [23]. According to the mentioned model, the risk of burnout also threatens professions different from socially relevant ones. The two-factor measurement model focuses on emotional exhaustion and depersonalization $[24,25]$, and the three-factor model $[26,27]$ assesses emotional exhaustion, depersonalization, and reduction of personal 
accomplishments. The four-factor model is similar to the three-item measurement, except for one of the measured dimensions being divided into two separate factors [28].

The processing models of burnout assessment consider burnout as a dynamic process, assuming that it is developing with time, i.e., from one phase or stage to another. An emotionally drained specialist tends to create emotional distance in his or her relationship with others to overcome the exhaustion. While experiencing the syndrome, however, one may develop a negative attitude towards own professional achievements. Since the profession of the anesthesiologist is highly responsible, the life of the patient may depend on the speed of reaction of the anesthesiologist. Therefore, anesthesiologists who are in a state of emotional burnout will show worse results than their healthy colleagues. It seems important to establish the reasons for possible emotional burnout in anesthesiologists. In this study, the attempt to establish what factors can influence the speed of burnout syndrome development in anesthesiologists, i.e., work experience, place of work, or gender, has been made. The authors assume that these factors may be significant in the formation of burnout syndrome in anesthesiologists. As a result, it can help identify risk groups and conduct corrective psychological work to prevent emotional burnout.

The following tasks have been set to achieve the aim, as mentioned earlier:

- study the prevalence and structure of the emotional burnout syndrome among anesthesiologists-resuscitators;

- explore the impact of gender differences in emotional burnout development;

- investigate the role of age in burnout development.

As the research objectives and approaches applied are relatively new, the decision was taken to utilize the less known methods for emotional burnout diagnosis (i.e., Boiko's emotional burnout measure) matching the research purpose.

\section{MATERIALS AND METHODS}

\section{Research Design}

The full composition of anesthesiologists working in hospitals of cities Astana and Akmola was assigned for the research. That was necessary for an adequate comparison of emotional burnout parameters, as all of them worked together and in the same conditions. Since this paper examines possible dependencies on gender, work experience, and workplace, it was recommended to include in the sample people working in the same conditions.

\section{Materials}

The study enrolled 144 anesthesiologistsresuscitators working in regional hospitals of Astana city (74 participants, aged $45.5 \pm 6.9$ years, on average) and Akmola city (70 participants, aged $49.5 \pm$ 7.5 years, on average). At the time of the survey, all participants were completely healthy and had no past medical history of chronic and hereditary diseases. In terms of gender distribution, $36 \%$ or 56 professionals were women, 31 of whom were occupied in the regional hospitals of Astana. The average age of women was $38.5 \pm 11.4$ years, and that of men -41.0 \pm 12.4 years. Work experience in anesthesiology ranged from 1 year to 20 years.

\section{Methods}

The testing was carried out at the beginning of the operation day. All anesthesiologists-resuscitators and nurses were about to participate in complicated surgical operations.

The study complies with the Protocol No. 5 issued by the Ethics Committee of the JSC "Astana Medical University. All the participants gave their consent to participate in this study.

The Boiko's Emotional Burnout Measure, an inventory designed to diagnose the burnout syndrome, was used to fulfill the aims of current research (Created by Vladimir Boiko in 1996).

The Boiko's Emotional Burnout Measure consists of an 84-item yes/no style questionnaire. Each item contributes to the overall score. Points in brackets are considered positive if the symbol in front of the item number is "+" and negative if the symbol is "-", respectively:

The feeling of being exposed to traumatic event or circumstances: +1(2), +13(3), +25(2), $-37(3),+49(10)$, $+61(5),-73(5)$

Dissatisfaction: $-2(3),+14(2),+26(2),-38(10),-50(5)$, $+62(5),+74(3)$ 
The feeling of being trapped in a cage: $+3(10),+15(5)$, $+27(2),+39(2),+51(5),+63(1),-75(5)$

Anxiety and depression: $+4(2),+16(3),+28(5),+40(5)$, $+52(10),+64(2),+76(3)$

Inadequate emotional response to certain stimuli: +5(5), -17(3), +29(10), +41(2), +53(2), +65(3), +77(5)

Emotional and moral disorientation: $+6(10),-18(3)$, $+30(3),+42(5),+54(2),+66(2),-78(5)$

Emotional suppression: $+7(2),+19(10),-31(2),+43(5)$, $+55(3),+67(3),-79(5)$

Reduction in productivity: $+8(5),+20(5),+32(2),-44(2)$, $+56(3),+68(3),+80(10)$

The feeling of emptiness: $+9(3),+21(2),+33(5),-45(5)$, $+57(3),-69(10),+81(2)$

Emotional disconnection: $+10(2), \quad+22(3), \quad-34(2)$, +46(3), +58(5), +70(5), +82(10)

Depersonalization: +11(5), +23(3), +35(3), +47(5), $+59(5),+72(2),+83(10)$

Psychosomatic disorders: +12(3), +24(2), +36(5), $+48(3),+60(2),+72(10),+84(5)$

Test results underlie the severity analysis of 12 symptoms that can be divided into three stages: Alarm Stage, Resistance Stage, and Exhaustion Stage. Symptom severity fluctuates between 0 and 30 points, with:

- 9 points and less indicating that no symptoms have developed;

- $\quad 10-15$ points indicating that the symptoms are developing;

16 points and more indicating the clear presence of the symptoms.

Symptoms that scored 20 points or more dominate the burnout stage or the syndrome in general.

For each stage, points scored in each symptom category are summed up. Hence, the total score for each stage can range from 0 to 120 . The factor within the stage that scored highest is the leading determinant of the professional burnout occurrence among respondents. To calculate the overall index, three total stage scores are summed up. The overall score can range from 0 to 360 .
By handling the semantic content and quantitative indicators calculated for various stages of the burnout syndrome development, it is possible to comprehensively characterize the respondent's personality, assess the adequacy of his or her emotional reactions during interpersonal interaction, and outline his or her individual preventive and psychocorrective measures.

\section{Statistical Analysis}

For the comparative analysis of the obtained data, the program Past v. 3.0 was used with subsequent transferring information in Microsoft Excel 2013(Microsoft Inc., USA) for database establishment. The calculated parameters characterizing the degree of emotional burnout were compared by applying correlation analysis. The methods of parametric analysis based on the normal distribution were applied, and Pearson's correlations between the compared groups were calculated for each of the three stages, including four features. The differences were defined as significant at $p \leq 0.05$. The relationship between personality traits was investigated through a hierarchical cluster analysis using Ward's method.

\section{RESULTS}

Statistics describing the prevalence of burnout symptoms as well as its stages, are presented in Figure 1.

From data in the above-given figure, it can be seen that the majority of participants are going through the second stage of emotional burnout with emotional lability, hysteria symptoms, and psychasthenia disturbances such as anxiety and suspiciousness. The moderate stress level they are experiencing is easy to deal with. Participants who reached exhaustion (twice as less as respondents at stage I) demonstrate the predominance of neurotic overcontrolling behavior, which manifests itself in the propensity to control one's emotions, follow the rules, and overestimate the severity of won condition. Respondents at stage III feel strong anxiety towards work and experience stress at a level higher than moderate. A small number of health care professionals (participants at stage I) standout with impulsiveness, tendency towards conflicts, and emotional lability. The signs of stress can be found at all stages, although it resembles a common problem at stage III that requires correction. A failure to cope with stress in the workplace is a good reason for the thorough analysis of professional life. Based on the 
above, a person is likely to emotionally collapse if he/she possesses traits such as emotionalism, selfdramatization, anxiety and suspiciousness, impulsiveness, propensity towards conflicts, and aggressiveness.

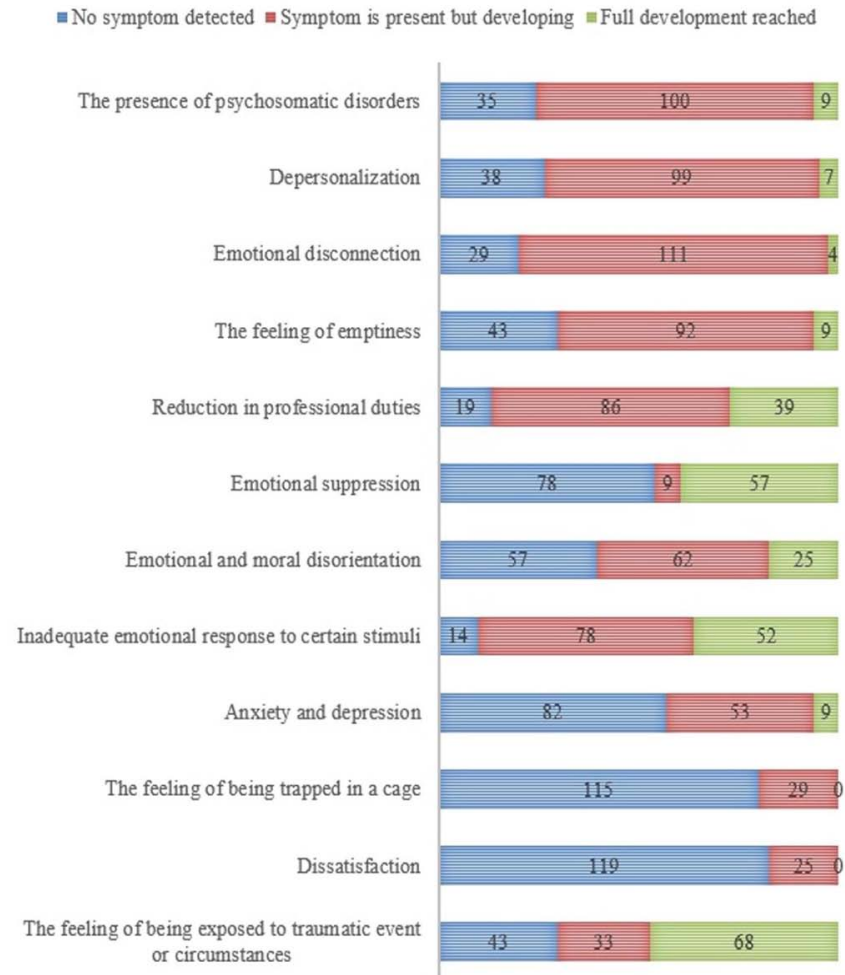

Figure 1: Burnout Prevalence, Stages of Development, and Symptoms.

Note (for Figures 1-4). The alarm stage includes the following categories: The feeling of being exposed to traumatic events or circumstances; Dissatisfaction; The feeling of being trapped in a cage; Anxiety and depression. The resistance stage includes: Inadequate emotional response to certain stimuli; Emotional and moral disorientation; Emotional suppression; Reduction in productivity. Exhaustion stage includes: The feeling of emptiness; Emotional disconnection; Depersonalization; The presence of psychosomatic disorder.

It is noteworthy that $47 \%$ of participants reported feeling as if they were exposed to traumatic events or circumstances, which means that they were aware of the traumatic effect of work they have performed.

Naturally, there is a high prevalence of early developing symptoms such as dissatisfaction and the feeling of being trapped, contributing to 83 and $80 \%$, respectively. An emotional transference effect occurs; the emotional energy generates in a negative form and is sent not into the world but onto the person generating it. In $57 \%$ of people, this condition progresses, and anxio-depressive symptoms appear.
A symptom such as inadequate emotional response $(54 \%)$ and Reduction in productivity $(60 \%)$ also prevail at the early stage of burnout syndrome development. In the case of the latter, respondents reported having numerous attempts to simplify or reduce own responsibilities requiring emotional efforts.

Symptoms in the exhaustion stage are even more frequent. Among them, the feeling of emptiness (64\%), emotional disconnection (77\%), depersonalization (68\%), and psychosomatic symptoms.

For the first stage, Pearson's significant correlation between the presence of symptoms and their development was 0.96 , and a negative correlation between the absence of symptoms and their development was -0.93 . The development of symptomatology in the first stage (alarm stage) is related to the presence of symptoms. At the second stage (resistance stage), this connection remained, and the values of correlations amounted to 0.94 and -0.91 , respectively. Thus, the first two stages initiate and form the process of emotional burnout. For the last stage (exhaustion stage), the following correlations were obtained: -0.98 for absence and presence of symptoms, 0.88 between symptom development and its presence, and -0.78 for the absence and development of a symptom. In the last stage, both positive and negative correlations between the signs increase.

In the primary health care facilities of Astana and Akmola cities, anesthesiologists suffer equally strongly from emotional burnout. The correlation value was 1.0 for all three stages. The same features were noticed when comparing the gender dependence of the emotional burnout expression signs: the correlation values were 0.70 . This suggests that a place of work facilitates the formation of emotional burnout stronger than gender. However, correlation values vary at different stages. Thus, for the first stage, the values of 0.88 were received, which assumes the absence of significant differences at the beginning of emotional burnout depending on gender, and for the second stage of burnout, these values have made 0.75 , i.e., there were some distinctions in the expression of emotional burnout between women and men. The third stage was characterized by the Pearson correlation value 0.06 if the significant factor was male and the value 0.91 - if female. Therefore, it can be concluded that women are significantly more exposed to emotional burnout at the last stage $(p \leq 0.001)$. 
Depending on the working experience, the following values were obtained. Anesthesiologists with up to 5 years of experience are significantly more likely to be stressed at the first stage (0.98), those with 5 to 10 years of experience are less likely to be stressed at the first stage (0.82), anesthesiologists with 10 to 15 years of experience are even less susceptible to be stressed at the first stage (0.78), and anesthesiologists with more than 15 years of experience are less likely to be stressed at the second stage (0.78). For the second stage, the correlations were insignificant, amounting to $0.95,0.95$, and 0.81 , respectively. Thus, a certain increase in the influence of stress factors on the condition of anesthesiologists can be observed, except for the group with more than 15 years of experience. Finally, at the third stage, Pearson's correlation values were $0.98,0.77,0.40$, and 0.40 , respectively. That allows concluding that employees working up to 5 years are most exposed to the risk of emotional burnout, but stress is also manifested at this stage in persons with 15 years of work experience.

Figure 2 provides statistics for the average rates of symptom prevalence and severity in the subgroups of anesthesiologists-resuscitators who work in the cities of Astana and Akmola.

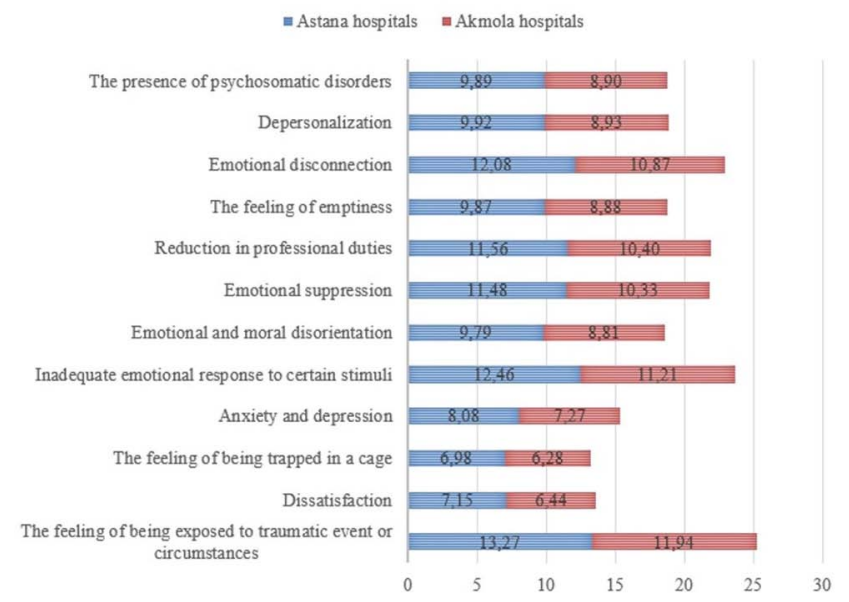

Figure 2: Subgroups in Astana Hospitals and Akmola Hospitals.

Data in Figure 2 indicate no significant differences in the severity of burnout symptoms between subgroups. Hence, the external factors influencing professional activity are either similar for both subgroups or do not significantly impact the emotional burnout development.

Figure 3 provides statistics for the average rates of symptom prevalence and severity in the subgroups of female and male respondents.

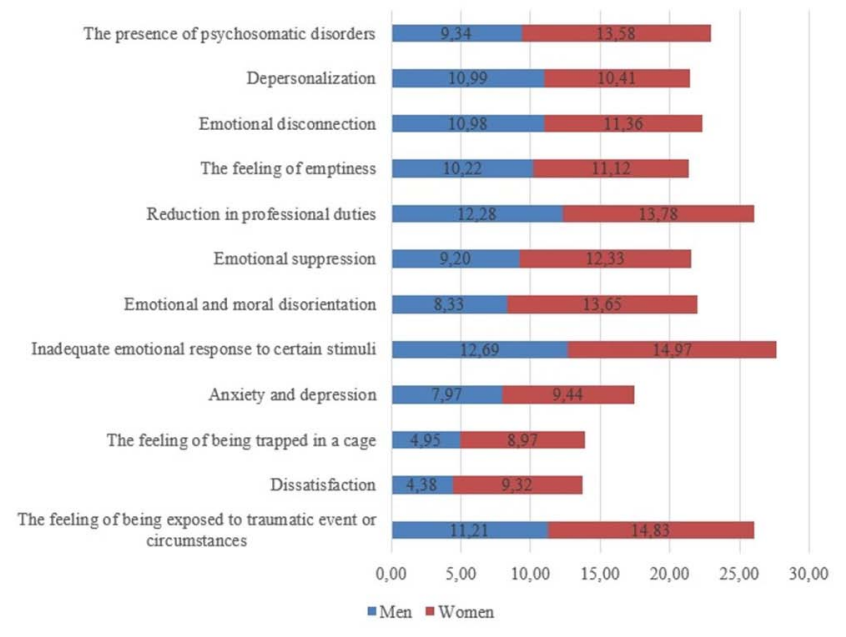

Figure 3: Comparative Parameters for Men and Women.

According to the data in Figure 3 , burnout symptoms occur at significantly higher frequency in women than in men. These include three alarm-stage symptoms such as the feeling of being in a stressful situation, dissatisfaction with oneself, and the feeling of being trapped; the resistance-stage symptoms include inadequate emotional response, emotional and moral disorientation, and emotional suppression.

Figure 4 provides statistics on connections to work experience. Data are divided into subgroups with anesthesiologists-resuscitators, who had 0 to 5,5 to 10,10 to 15 , and 15 to 20 years of work experience at the moment of research.

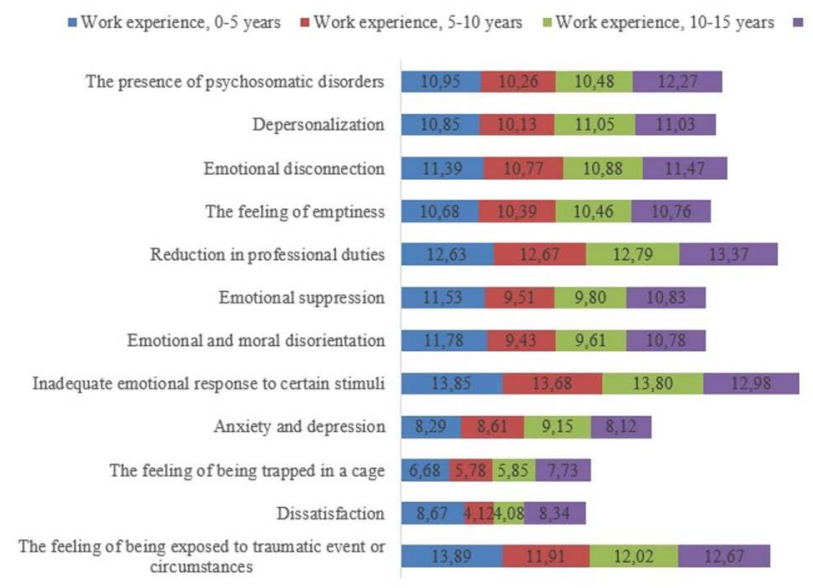

Figure 4: Parameters across Different Work Experience Groups.

The frequency and severity of alarm-stage symptoms (i.e., the feeling of being in a stressful situation, dissatisfaction with oneself, and the feeling of being trapped) seem to be distributed over subgroups in a specific way. The maximum scores were found in 
the first subgroup of respondents with work experience under 5 years. In the second subgroup (with experience ranges between 5 and 10 years), frequency and severity scores are significantly lower. In the third subgroup, an upward trend was observed, whilst the fourth subgroup demonstrated results higher than in the second subgroup but lower than in the first subgroup. In modern literature, young anesthesiologists are reported to be especially vulnerable to burnout syndrome. Using Ward's clustering method, a link between the personality characteristics of participants was established (Figure 5).

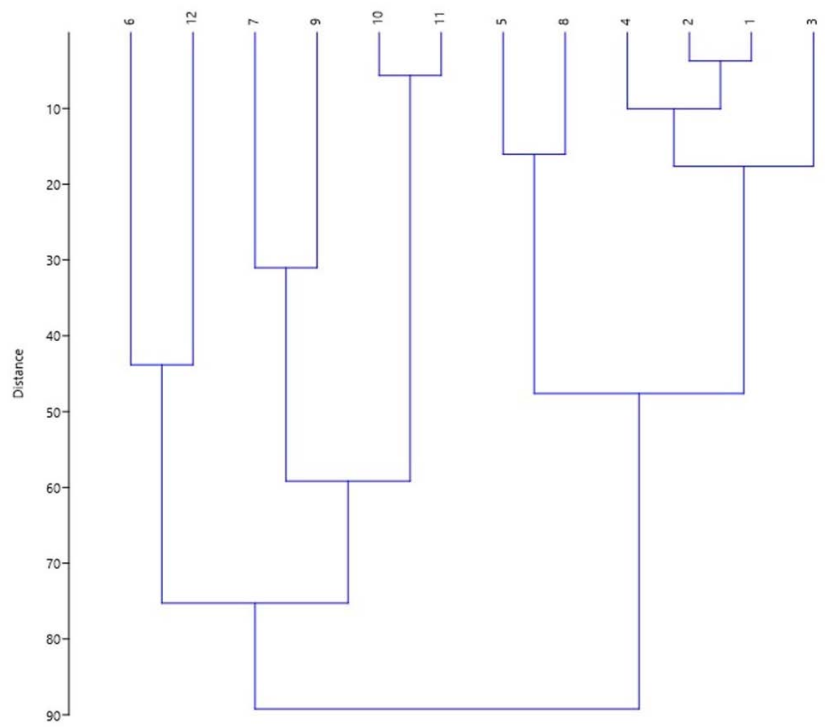

Figure 5: Dendrogram for Personality Traits among Participants in the Study.

Note (for Figures 4-8). 1 - The presence of psychosomatic disorder; 2 - Depersonalization; 3 - Emotional disconnection; 4 - The feeling of emptiness; 5 - Reduction in productivity; 6 - Emotional suppression; 7 - Emotional and moral disorientation; 8 - Inadequate emotional response to certain stimuli; 9 - Anxiety and depression; 10 - The feeling of being trapped in a cage; 11 - Dissatisfaction; 12 - The feeling of being exposed to traumatic event or circumstances.

The first cluster consists of symptoms 1-4, which correspond to the alarm stage of burnout syndrome development, and symptoms 5 and 8 , which correspond to the resistance stage. These signs make up the most likely scenario of response to stress at work, which manifests in emotional instability, destructive changes in productivity, and inadequate response to stimuli. The second cluster includes symptoms $7,9,10$, and 11 , of which symptoms 10 and 11 correspond to the exhaustion stage of burnout syndrome development. Thusly, an individual, who experiences negative changes in the structure of personality under the influence of professional activity, may feel dissatisfaction and have the sense of being constricted. Finally, the third cluster consists of symptoms 6 (resistance stage) and 12 (exhaustion stage), which correspond to emotional suppression and distressing experience. These signs constitute an alternative scenario of burnout.

Personality traits of specialists employed by hospitals in Astana and Akmola were distributed as follows (Figure 6).

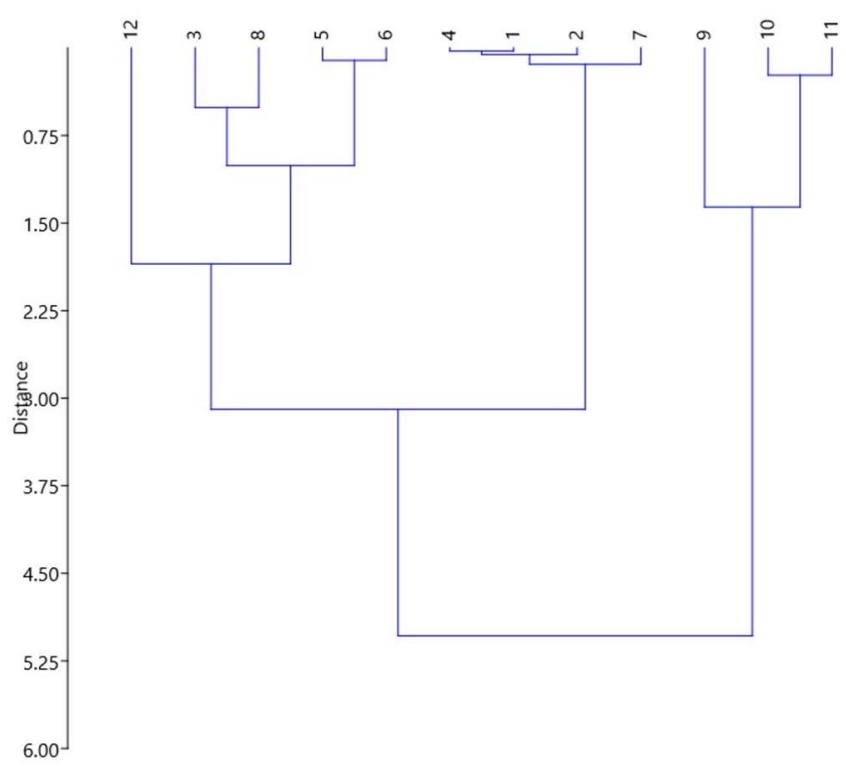

Figure 6: Dendrogram for Personality Traits among Hospitalemployed Specialists (Astana, Akmola).

The first cluster consisted of symptoms 9,10 , and 11 , the second cluster $1,4,2$, and 7 , and the third cluster was composed of other symptoms. As can be seen, the first cluster represents the most severe stage of burnout, while the second and third clusters portray the light and moderate cases. Thus, there are three possible scenarios of burnout relative to medical specialists: severe, moderate, and light. There were no gender differences in the structure of burnout (Figure 7)

As shown in Figure 7, there are four likely scenarios of emotional burnout, which can be associated with both men and women. The first cluster includes symptoms 5,8 , and 12 , which indicate that destructive changes in productivity can make individuals believe that their work is too intense. The consequence is the feeling of being exposed to traumatic stress. The second cluster consisting of symptoms 2-4 (depersonalization, emotional disconnection, and the feeling of emptiness, respectively) represents the light case of burnout, where depersonalization causes the feeling of emptiness. The third cluster with symptoms 1 and 6-7 reflects the situation where the psychosomatic 
disorder is the cause of emotional suppression and disorientation. The fourth cluster with symptoms 9-11 corresponds to the most severe scenario (exhaustion). There were differences in the results of clustering analysis associated with the years of working experience (Figure 8).

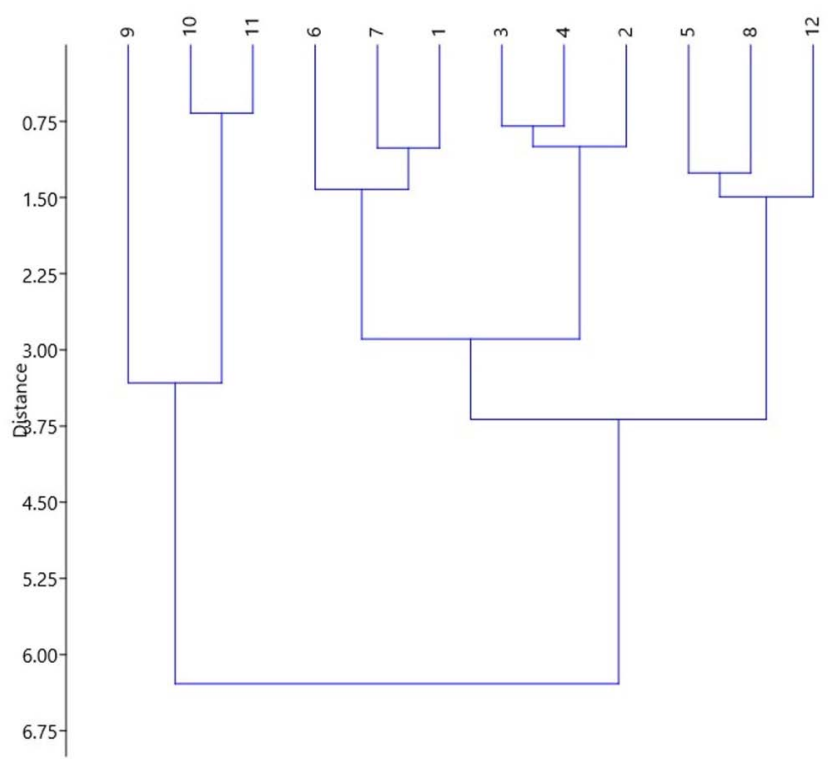

Figure 7: Dendrogram for Personality Traits among Male and Female Participants.

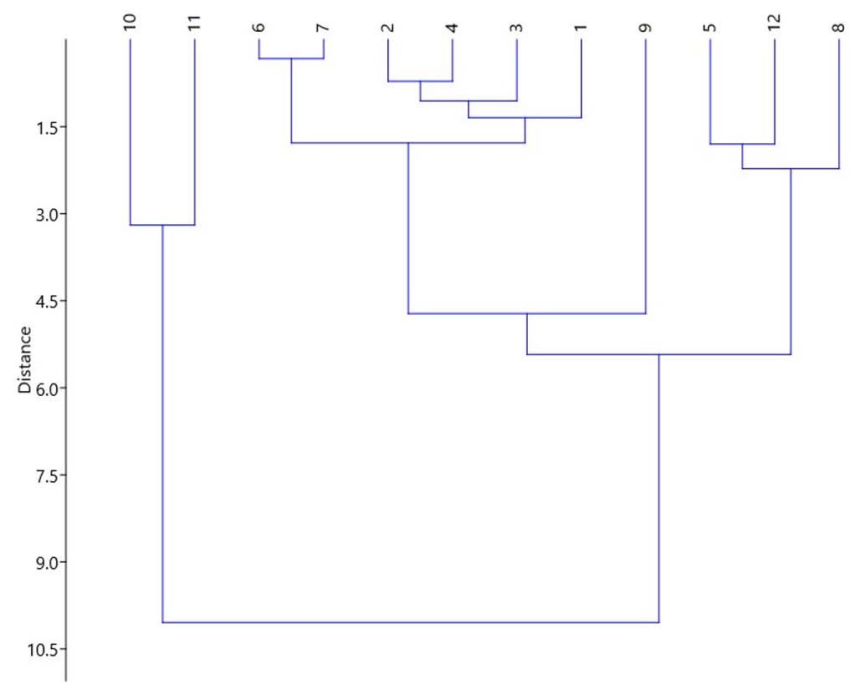

Figure 8: Dendrogram based on Cluster Analysis Showing the Relationship between Burnout Symptoms and Years of Working Experience.

Based on data in Figure 8, burnout symptoms can vary in type and severity between specialists with different years of working experience. For instance, individuals who have more than 15 years of working experience may lose productivity (5), show an inadequate response to stimuli (8), and feel traumatic stress (12). By contrast, individuals with up to 5 years of working experience are likely to encounter a diverse range of destructive changes, such as psychosomatic disorders, depersonalization, emotional disconnection, the feeling of emptiness, emotional suppression, moral disorientation, anxiety, and even depression. In this case, exhaustion can be the cause of depression. Finally, individuals with 5 to 10 years of experience are likely to feel caged and dissatisfied with their lives.

\section{DISCUSSION}

When examining emotional burnout symptoms for each distinguished stage, a connection between personality traits and the state of health in employees was established. Character traits are internal factors that contribute to the emergence and formation of emotional burnout syndrome.

The overall job satisfaction level among anesthesiologists in this study is comparable to findings in other studies [29]. Sanfilippo et al. [14] indicated that burnout syndrome varies in prevalence among anesthesiologists from $10 \%$ to $59 \%$. Besides, risk factors include years of work, the presence of children, and the work's intensity. In this study, professional experience is considered one of the main risk factors, which is confirmed by the results of these authors. According to other data [15], the gender distribution among anesthesiologists with burnout syndrome is approximately equal $-51 \%$ of men and $49 \%$ of women. In the data of our research, women are more likely to be burned out emotionally. These parameters may vary greatly from sample to sample. The risk age range is between 30 and 50 years, which corresponds to the age group in this study, while other data show that men are more likely to be burned out [30].

The present results demonstrate that anesthesiologists working longer hours are more vulnerable to burnout and likely to be dissatisfied with different life aspects. Other researchers confirm that burnout is strongly influenced by attitudes within the family [21].

Numerous studies prove that burnout occurs in several stages:

1. Dealing with pressure caused by a need to adapt to situational business demands.

2. Feeling strong sensations and experiencing stress. 
3. Responding to three major symptom categories of stress (physiological, affective-cognitive, and behavioral).

4. Emotional burnout as a multifaceted experience of chronic psychological stress.

In contrast, Greenberg [12] defined burnout as a progressive five-step process.

1. Honeymoon. Professional activity starts to bring less pleasure, and the employee becomes less energetic.

2. The Lack of Fuel. Feelings of tiredness and apathy appear. This may cause sleep disturbances.

3. Chronic Symptoms. Excessive work without rest, especially in workaholics, increases sensitivity to somatic diseases. The worker constantly feels like he/she lacks time.

4. Crisis. Symptoms become critical with partial or complete loss of labor efficiency.

5. Breaking Down the Walls. Physical and psychological problems become embedded in life and may cause life-threatening diseases to develop.

\section{CONCLUSION}

The present research describes features characterizing the structure and dynamics of the emotional burnout syndrome development in anesthesiologist-resuscitators. Findings demonstrate that women are more likely to have emotional burnout syndrome compared to men. Also, burnout occurrence and development are attributed to the work experience.

\section{REFERENCES}

[1] Downey RL, Farhat T, Schumann R. Burnout and coping amongst anesthesiologists in a US metropolitan area: a pilot study. Middle East J Anaesthesiol 2012; 21(4): 529-34.

[2] Oliveira RV, Schneider V, Bonafé FSS, Maroco J, Campos JADB. Occupational characteristics and Burnout Syndrome in Brazilian correctional staff. Work 2016; 55(1): 215-23. https://doi.org/10.3233/WOR-162367

[3] Mata DA, Ramos MA, Bansal N, et al. Prevalence of depression and depressive symptoms among resident physicians: A systematic review and meta-analysis. JAMA 2015; 314: 2373-83.

https://doi.org/10.1001/jama.2015.15845

[4] Wurm W, Vogel K, Holl A, et al. Depression-burnout overlap in physicians. PLoS One 2016; 11: e0149913. https://doi.org/10.1371/journal.pone.0149913
Parola V, Coelho A, Cardoso D, Sandgren A, Apóstolo J. Prevalence of burnout in health of professionals working in palliative care. JBI Database Syst Rev Implement Rep 2017; 15: 1905-33.

https://doi.org/10.11124/JBISRIR-2016-003309

[6] Dias FM, Santos JF de C, Abelha L, Lovisi GM. Occupational stress and professional exhaustion syndrome (burnout) in workers from the petroleum industry: A systematic review. Rev Bras Saúde Ocup 2016; 41: 4 https://doi.org/10.1590/2317-6369000106715

[7] Koutsimani P, Montgomery A, Georganta K. The relationship between burnout, depression, and anxiety: A systematic review and meta-analysis. Front Psychol 2019; 10: 1-19. https://doi.org/10.3389/fpsyg.2019.00284

[8] Eslava-Schmalbach J, Garzón-Orjuela N, Gómez-Restrepo C. Prevalence and Factors Associated with Burnout Syndrome in Colombian Anesthesiologists. Int J Prev Med 2020; $11: 5$.

https://doi.org/10.4103/ijpvm.IJPVM 15018

[9] Kansoun Z, Boyer L, Hodgkinson M, Villes V, Lançon C, Fond G. Burnout in French physicians: A systematic review and meta-analysis. J Affect Disord 2018; 246: 132-47. https://doi.org/10.1016/j.jad.2018.12.056

[10] Perlman B, Hartman EA. Burnout: Summary and Future Research. Hum Relations SAGE Publications 1982; 35(4): 283-305. https://doi.org/10.1177/001872678203500402

[11] Burisch M. In search of theory: Some ruminations on the nature and etiology of burnout. In: Schaufeli WB, Maslach C, Marek T, editors. Series in applied psychology: Social issues and questions. Professional burnout: Recent developments in theory and research. Abingdon: Taylor \& Francis 1993; p. 75-93. https://doi.org/10.4324/9781315227979-7

[12] Greenberg JS. Comprehensive stress management, 13th ed. New York: McGraw-Hill; 2013.

[13] Korczak D, Kister C, Huber B. Differentialdiagnostik des Burnout-Syndroms. Cologne: Deutsches Institut für Medizinische Dokumentation und Information; 2010.

[14] Sanfilippo F, Noto A, Foresta G, et al. Incidence and Factors Associated with Burnout in Anesthesiology: A Systematic Review. BioMed Res Int 2017; 1: 8648925 https://doi.org/10.1155/2017/8648925

[15] Barbosa FT, Eloi RJ, dos Santos LM, Leão BA, de Lima FJC, de Sousa-Rodrigues CF. Correlation between weekly working time and burnout syndrome among anesthesiologists of Maceió-AL. Braz J Anesthesiol 2017; 67(2): 115-121. https://doi.org/10.1016/j.bjan.2015.06.001

[16] Yumashev AV, Gorobets TN, Admakin OI, Kuzminov GG Nefedova IV. Key aspects of adaptation syndrome development and anti-stress effect of mesodiencephalic modulation. Indian J Sci Technol 2016; 9(19): 93911. https://doi.org/10.17485/ijst/2016/v9i19/93911

[17] Boiko VV. The syndrome of "emotional burnout" in professional communication. St. Petersburg: Piter; 1999.

[18] Boiko VV. The energy of emotions. Moscow: Filin; 2004.

[19] Boiko VV. Psychoenergetics. St. Petersburg: Piter; 2008.

[20] Selye H. Studies on an adaptation syndrome. Moscow: Medgiz; 1960.

[21] De Oliveira GSJr, Almeida MD, Ahmad S, Fitzgerald PC, McCarthy RJ. Anesthesiology residency program director burnout. J Clin Anesth 2011; 23: 176-82.

https://doi.org/10.1016/j.jclinane.2011.02.001

[22] West CP, Dyrbye LN, Satele DV, Sloan JA, Shanafelt TD. Concurrent validity of single-item measures of emotional exhaustion and depersonalization in burnout assessment. $\mathrm{J}$ Gen Intern Med 2012; 27: 1445-52. https://doi.org/10.1007/s11606-012-2015-7 
[23] Pines AM, Aronson E. Burnout: from tedium to personal growth. Washington DC: Taylor \& Francis; 1993.

[24] van Dierendonck D, Schaufeli WB, Buunk BP. Toward a process model of burnout: Results from a secondary analysis. Eur J Work Organ Psychol 2001; 10(1): 41-52. https://doi.org/10.1080/13594320042000025

[25] van Dierendonck D, Schaufeli W, Sixma H. Burnout among general practitioners: a perspective from equity theory. J Soc Clin Psychol 1994; 13(1): 86-100. https://doi.org/10.1521/jscp.1994.13.1.86

[26] Frith GH, Mims A. Burnout among special education paraprofessionals. Teach Except Child 1985; 17(3): 225-7. https://doi.org/10.1177/004005998501700312
[27] Maslach C, Jackson S. MBI: Maslach Burnout Inventory. Manual Res. Palo Alto, CA: Consulting Psychologists Press; 1986.

[28] Iwanicki EF, Schwab RL. Across-validation of the Maslach Burnout Inventory. Educ Psychol Meas 1981; 41: 1167-74. https://doi.org/10.1177/001316448104100425

[29] Abut YC, Kitapcioglu D, Erkalp K, et al. Job burnout in 159 anesthesiology trainees. Saudi J Anaesth 2012; 6(1): 46. https://doi.org/10.4103/1658-354X.93059

[30] Magalhães E, Oliveira ÁCMDS, Govêia CS, Ladeira LCA Queiroz DM, Vieira CV. Prevalence of burnout syndrome among anesthesiologists in the Federal District. Rev Bras Anestesiol 2015; 65(2): 104-110. https://doi.org/10.1016/j.bjan.2013.07.016

Received on 28-09-2020

DOI: https://doi.org/10.6000/2292-2598.2020.08.04.12

(c) 2020 Kokoshko et al.; Licensee Lifescience Global.

This is an open access article licensed under the terms of the Creative Commons Attribution Non-Commercial License (http://creativecommons.org/licenses/by-nc/3.0/) which permits unrestricted, non-commercial use, distribution and reproduction in any medium, provided the work is properly cited. 